Check for updates

Cite this: RSC Adv., 2018, 8, 28480

Received 12th July 2018

Accepted 2nd August 2018

DOI: 10.1039/c8ra05957h

rsc.li/rsc-advances

\section{Electrospinning preparation of a large surface area, hierarchically porous, and interconnected carbon nanofibrous network using polysulfone as a sacrificial polymer for high performance supercapacitors $\dagger$}

\begin{abstract}
Wenyu Wang, ${ }^{* a}$ Hongjie Wang, ${ }^{a}$ He Wang, ${ }^{a}$ Xin Jin, ${ }^{b}$ Jialu Li ${ }^{a}$ and Zhengtao Zhu (DD *ac
Carbon nanofibrous mats (CNFMs) are prepared by electrospinning of blended precursor of polyacrylonitrile and polysulfone (PSF) followed by pre-oxidation stabilization and carbonization. Addition of PSF as a sacrificial polymer leads to CNFMs with high surface area, large numbers of micropores and mesopores, good degree of carbonization, and interconnected fibrous network, due to the high decomposition temperature, release of $\mathrm{SO}_{2}$ during decomposition, and large amount of carbon residue of PSF during carbonization. The electrochemical characterization shows that the CNFM electrode has a specific capacitance of $272 \mathrm{~F} \mathrm{~g}^{-1}$ at a current density of $1 \mathrm{~A} \mathrm{~g}^{-1}$ with $74 \%$ of the specific capacitance retained at $50 \mathrm{~A} \mathrm{~g}^{-1}$ in $2.0 \mathrm{M} \mathrm{KOH}$ electrolyte. The CNFM electrodes have excellent cycling durability with $100 \%$ capacitance retention after 10000 cycles.
\end{abstract}

\section{Introduction}

Electrochemical energy storage systems are crucially important in the development of renewable, sustainable, and eco-friendly energy technologies because of the intermittent nature of the available renewable energy sources. Among various electrochemical energy storage devices, supercapacitors have attracted wide attention in recent years because of their fast chargedischarge rate, high power density, excellent cycle durability, and low fabrication/operation cost. ${ }^{1}$ In supercapacitors, particularly in the electrochemical double layer capacitors (EDLCs), the electrode materials largely determine the performance of the devices; therefore, considerable efforts have been devoted to develop advanced materials for supercapacitor electrode..$^{2-5}$ Carbon nanofibrous mats (CNFMs) prepared from electrospinning have shown great potential as electrode materials for supercapacitors. ${ }^{6-9}$ For high performance supercapacitors, the ideal CNFMs need to have (1) large surface areas, (2) hierarchical pore structures, and (3) high electrical conductivity. In a supercapacitor, the electric charges are

${ }^{a}$ State Key Laboratory of Separation Membranes and Membrane Processes, School of Textiles, Tianjin Polytechnic University, Tianjin 300387, China. E-mail: wwy-126.com ${ }^{b}$ School of Materials Science and Engineering, Tianjin Polytechnic University, Tianjin 300387, China

${ }^{c}$ Department of Chemistry and Applied Biological Science, South Dakota School of Mines and Technology, Rapid City, SD 57701, USA. E-mail: zhengtao.zhu@sdsmt.edu $\dagger$ Electronic supplementary information (ESI) available: SEM images of precursor nanofibrous mats; pore size distribution; XPS, and DSC of carbon nanofibrous mats; GDC curves of carbon nanofibrous mats; comparison of supercapacitors based electrospun nanofibrous materials. See DOI: 10.1039/c8ra05957h adsorbed and stored on the surface of the electrode, therefore, porous structures with large surface areas are required to have high charge storage capacity. Additionally, high surface areas may not necessarily result in high performance, since not all pores can be utilized for charge storage; hierarchical pore structures ranging from micropore (0.5-2 $\mathrm{nm})$, mesopore (2-50 $\mathrm{nm})$, and macropore $(>50 \mathrm{~nm})$ are desired for effective electrolyte ion transport and charge storage. Furthermore, high electric conductivity of CNFMs reduces the internal resistance of the supercapacitors.

CNFMs prepared by electrospinning and carbonization typically have limited surface area and conductivity. Many works have focused on preparation of CNFMs with high surface areas and desired porous structures. One approach is to use blended polymer precursor consisting of a carbon source polymer and a sacrificial polymer as spin dope to increase the surface areas and to control the pore sizes..$^{\mathbf{1 0 - 1 4}}$ The common sacrificial polymer (e.g., polymethylmethacrylate and polyvinylpyrrolidone) degrades completely without carbon residues at temperatures well below carbonization temperature, leading to porous structures of CNFMs. In general, the nanofibers produced by this method need to be further activated to increase the surface areas; and the pores produced are largely mesopores, which may not all contribute to the charge storage. The conductivity of CNFMs may be improved by increasing the carbonization temperature, which increases the graphitic carbon contents of individual nanofiber. To further improve the conductivity of CNFMs, interconnected fibrous networks of CNFMs are desired. Few studies have been reported to prepare the interconnected CNFMs, particularly the ideal electrode 
material of the interconnected CNFMs with high surface areas and hierarchical porous structures. ${ }^{15-17}$

Recently, we reported preparation of carbon nanofibrous mat by carbonization of electrospun nanofibers of polyacrylonitrile (PAN) and polysulfone (PSF) blends. ${ }^{18}$ As an in situ activation agent, PSF helped formation of CNFMs with increased mesopore content, specific surface area, graphitization degree, and conductivity of CNFMs. Slurries of CNFMs, carbon black, and poly(tetrafluoroethylene) were made into electrodes for twoelectrode symmetric supercapacitor. The supercapacitor showed good performance, cycling stability, and energy capacity.

Herein, we report our further studies on the electrospun CNFMs prepared from the blended polymer precursor composed of PAN and PSF. Based on the morphological and structural characterization, thermal analysis, surface area and porosity measurements, we propose a detailed mechanism for formation of high surface area, microporous and mesoporous structure, well interconnected carbon nanofibrous network with good graphitic carbon contents of the PAN/PSF-based CNFMs. Furthermore, the electrochemical performance of the supercapacitor electrode prepared by the freestanding CNFMs without further activation and additives is characterized under high charge/discharge rate. The result shows a high specific capacitance of $\sim 272 \mathrm{~F} \mathrm{~g}^{-1}$ at $1 \mathrm{~A} \mathrm{~g}^{-1}$ current density and $\sim 200 \mathrm{~F}$ $\mathrm{g}^{-1}$ at a fast charge rate of $50 \mathrm{~A} \mathrm{~g}^{-1}$, demonstrating excellent electrochemical properties under fast charge/discharge conditions. The electrodes retain excellent cycling durability with $100 \%$ capacitance retention at $1 \mathrm{~A} \mathrm{~g}^{-1}$ rate after 10000 cycles.

\section{Experimental}

\subsection{Materials}

Polyacrylonitrile (PAN, $\left.M_{\mathrm{w}}=90000\right)$ was purchased from Spectrum Co., Ltd, Shanghai. Polysulfone (PSF, $M_{\mathrm{w}}=55000$ ) was obtained from Solvay Chemical Co., Ltd. $N, N$-Dimethylformamide (DMF) was purchased from Kemiou Chemical Reagent Co., Ltd, Tianjin. Potassium hydroxide $(\mathrm{KOH}$, purity $\geq$ 85\%) was obtained from Fengchuan Chemical Reagent Science and Technology Co., Ltd, Tianjin. Deionized water was used in all experimental processes.

\subsection{Preparation of CNFMs}

PSF and PAN were dissolved simultaneously in DMF to form a homogeneous precursor solution. The concentration of PAN and PSF was $8.0 \mathrm{wt} \%$ and $2.0 \mathrm{wt} \%$, respectively, thus there was $20 \mathrm{wt} \%$ of PSF in the obtained PSF/PAN precursor nanofibers. For the electrospinning process, the spin dope was loaded in a syringe spinneret, and an applied voltage between the stainlesssteel needle of the syringe and the grounded collector was set at $20 \mathrm{kV}$; the distance between the spinneret and the collector was $18 \mathrm{~cm}$ and the flow rate was set at $16.67 \mu \mathrm{L} \mathrm{min}^{-1}$. The as-spun PSF/PAN nanofibrous mat (denoted as NFM-20) was preoxidized at $280{ }^{\circ} \mathrm{C}$ in air for 1 hour after ramping the temperature to $280^{\circ} \mathrm{C}$ at a rate of $2{ }^{\circ} \mathrm{C} \mathrm{min}^{-1}$; then the pre-oxidized NFM20 was carbonized at $800{ }^{\circ} \mathrm{C}$ in nitrogen for 2 hours after the temperature was ramped to $800{ }^{\circ} \mathrm{C}$ at a rate of $5{ }^{\circ} \mathrm{C} \mathrm{min}^{-1}$. The obtained carbon nanofibrous mat from NFM-20 was denoted as CNFM-20. The electrospun PAN nanofibrous mat (denoted as NFM-0) without PSF and the subsequent carbon nanofibrous mat (CNFM-0) were prepared following the same procedure.

\subsection{Characterization}

The surface morphology of the nanofibers was observed using scanning electronic microscope (SEM, Gemini SEM500) and high-resolution transmission electron microscopy (HRTEM, JEM-2100F). The degree of carbonization was evaluated by X-ray diffraction spectrometer (XRD, D8 DISCOVER). Raman spectra were collected by a Raman spectrometer (Raman, XploRA PLUS) with a $532 \mathrm{~nm}$ excitation laser. The thermogravimetric data was acquired using STA449F3 analyzer at a scanning rate of $10^{\circ} \mathrm{C} \mathrm{min}^{-1}$ under nitrogen atmosphere. Thermal behaviors of the samples were characterized by differential scanning calorimeter (DSC, DSC 200F3) at a heating rate of $10^{\circ} \mathrm{C} \mathrm{min}^{-1}$ under nitrogen atmosphere. The surface chemical composition was analyzed by X-ray photoelectron spectroscopy (XPS, K-alpha) equipped with a $165 \mathrm{~mm}$ hemispherical electron energy analyzer. BET surface areas of the samples were determined using the nitrogen adsorption method on Autosorb-iQ-C. The electrical conductivity of the CNFs was measured using a semiconductor powder resistivity tester (ST-2722).

\subsection{Fabrication and electrochemical characterization of CNFMs based supercapacitor}

The freestanding CNFM was pressed directly on a circular nickel foam (diameter: $1.2 \mathrm{~cm}$; loading: $\sim 2 \mathrm{mg}$ ) to be used as an electrode. For the three-electrode electrochemical measurement, Pt foil was used as the counter electrode, and $\mathrm{Hg} / \mathrm{HgO}$ was used as the reference electrode. The $2.0 \mathrm{M} \mathrm{KOH}$ aqueous solution was employed as the electrolyte. The cyclic voltammetry $(\mathrm{CV})$ was measured between 0 and $-1.0 \mathrm{~V}$ at a scan rate of $10 \mathrm{mV} \mathrm{s}^{-1}$, and the galvanostatic charge/discharge (GCD) curves were collected at various current densities ranging from $1.0 \mathrm{~A} \mathrm{~g}^{-1}$ to $50 \mathrm{~A} \mathrm{~g}^{-1}$ by an electrochemical workstation (Zahner, Zennium 2.0+CIMPS-2). The retention rate of electrodes was evaluated at a current density of $1.0 \mathrm{~A} \mathrm{~g}^{-1}$ for 10000 cycles using a supercapacitor test system (LANHE, CT2001A). The electrochemical impedance spectroscopy (EIS) of the electrodes was measured at the open circuit voltage with an AC amplitude of $5 \mathrm{mV}$ over the frequency range of $10 \mathrm{mHz}$ to $10 \mathrm{kHz}$.

Two-electrode supercapacitor was fabricated using two identical CNFM electrodes with a piece of $\mathrm{PP} / \mathrm{PE}$ composite as separator and 2.0 $\mathrm{M} \mathrm{KOH}$ aqueous solution as electrolyte. The cyclic voltammetry (CV) was measured between 0 and $1.0 \mathrm{~V}$ at various scan rate, and the galvanostatic charge/discharge (GCD) curves were collected at various current densities ranging from $0.25 \mathrm{~A} \mathrm{~g}^{-1}$ to $50 \mathrm{~A} \mathrm{~g}^{-1}$ by an electrochemical workstation (Zahner, Zennium 2.0+CIMPS-2).

\section{Results and discussion}

SEM images of the as-prepared precursor nanofibrous mats, NFM-0 and NFM-20, are shown in Fig. S1. $\dagger$ Both NFM-0 and 
NFM-20 have similar morphology with smooth and randomly overlaid nanofibers. For individual nanofiber, the diameter of the nanofiber is uniform. No beads are observed in the SEM images of both samples. SEM images of CNFM-0 with different magnifications are shown in Fig. 1a, $\mathrm{a}^{\prime}$ and $\mathrm{a}^{\prime \prime}$. Compared to the precursor PAN nanofibrous mat (i.e. NFM-0), the nanofibers of CNFM-0 are slightly curly, likely due to the shrinkage during carbonization. The average diameter of CNFM-0 is $190 \pm 60 \mathrm{~nm}$, smaller than that of NFM-0. The morphology of CNFM-20, which is carbonized from the PSF/PAN precursor fibrous mat (i.e. NFM-20), is shown in Fig. $1 \mathrm{~b}, \mathrm{~b}^{\prime}$ and $\mathrm{b}^{\prime \prime}$. The diameter of CNFM-20 is $115 \pm 65 \mathrm{~nm}$, much smaller than that of CNFM-0. Compared with the nanofibers of CNFM-0, the individual nanofiber of CNFM-20 has more curly structure and nonuniform diameter. More importantly, the nanofibers of CNFM-20 are weld together at the fiber junction points to form an interconnected fibrous network (Fig. $1 b^{\prime}$ ), whereas no welding to fuse the nanofibers at the fiber junction points for the PANderived CNFM-0 (Fig. 1 $\mathrm{a}^{\prime}$ ). Fig. 1 $\mathrm{a}^{\prime \prime}$ and $\mathrm{b}^{\prime \prime}$ show the high magnification cross-sectional SEM images of the nanofibers in CNFM-0 and CNFM-20, respectively; porous structures are observed ubiquitously throughout the nanofibers of the CNFM20. The HRTEM image of the CNFM-20 in Fig. S2† shows that the CNFM-20 nanofibers have good microporous structures.

The pore information of CNFM-0 and CNFM-20 including the BET specific surface area, microporous and mesoporous volumes is summarized in Table 1 . The PAN based CNFM-0 has specific surface area of $96 \mathrm{~m}^{2} \mathrm{~g}^{-1}$; and the CNFM-20 from PSF/ PAN has specific surface area of $763 \mathrm{~m}^{2} \mathrm{~g}^{-1}$, about 8 times higher than that of CNFM-0. As shown in Fig. 2a, CNFM-20 have higher nitrogen adsorbed volume at low pressure, indicating that the PSF/PAN based CNFM-20 has a better interfacial adsorption capacity than the PAN based CNFM-0. The surface
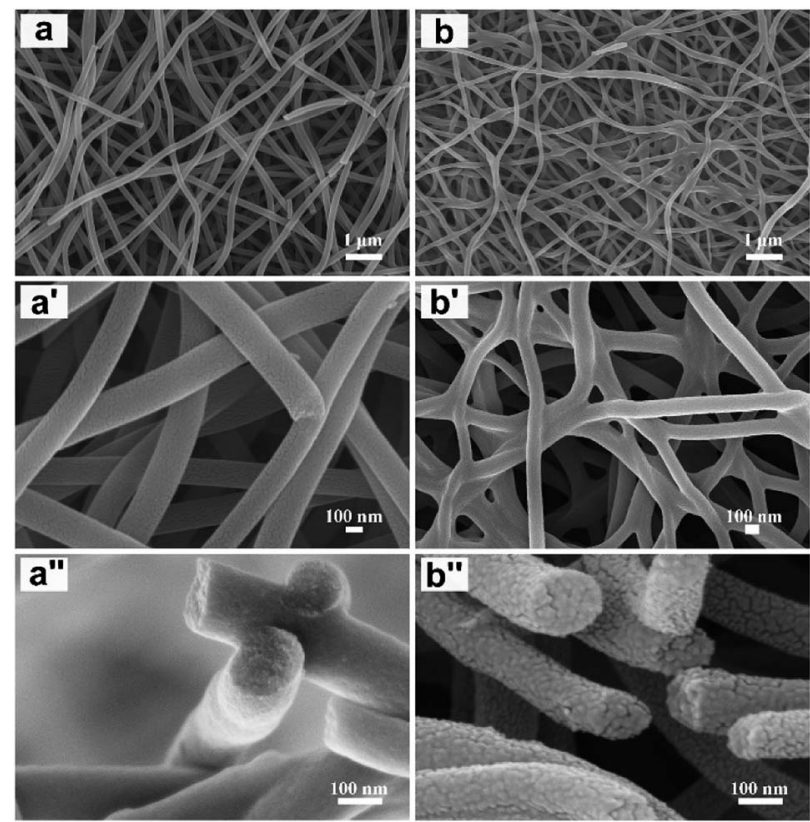

Fig. 1 SEM images of CNFM-O (a, $a^{\prime}$ and $\left.a^{\prime \prime}\right)$ and CNFM-20 (b, $b^{\prime}$ and $b^{\prime \prime}$ ). area of the micropore in CNFM-20, measured by Langmuir method, is about 5 times higher than that of CNFM-0. The mesopore volume distribution of CNFM-20 and CNFM-0 is showed in Fig. S3, $\uparrow$ and the result clearly indicates that CNFM20 has higher mesopore volume than CNFM-0. Overall, addition of PSF results in much higher surface areas and better hierarchical pore structures in CNFM-20. Fig. 2b shows the XRD patterns of CNFM-0 and CNFM-20. Both CNFMs have a strong diffraction peak at $2 \theta=22^{\circ}$ and a weak diffraction peak at $2 \theta=$ $16^{\circ}$, corresponding to the graphitic crystallite plane (002) and the PAN crystallite plane (100), respectively. ${ }^{19,20}$ Presence of the (100) peak indicates incomplete carbonization, which is likely due to the relative low carbonization temperature of $800{ }^{\circ} \mathrm{C}^{21}$ With addition of PSF, the (100) peak becomes weaker and the (002) peak becomes stronger, indicating increase of the amount of the graphitic carbon in CNFM-20. Fig. 2c shows the Raman spectra of CNFM-0 and CNFM-20. The spectra of both samples show the typical $\mathrm{G}$ band $\left(1585 \mathrm{~cm}^{-1}\right)$ and $\mathrm{D}$ band $\left(1350 \mathrm{~cm}^{-1}\right)$ for carbonaceous materials. The $\mathrm{G}$ band is related to the ordered graphitic carbon, whereas the $\mathrm{D}$ band is related to the amorphous and disordered carbon. The spectrum of CNFM-20 shows a much stronger $\mathrm{G}$ band compared to the spectrum of CNFM-0, indicating higher content of the $\mathrm{sp}^{2}$ hybridized graphitic carbon in CNFM-20. The degree of carbonization is also evaluated by the intensity ratio $(R)$ of the $\mathrm{D}$ and $\mathrm{G}$ bands (i.e. $\left.R=I_{\mathrm{D}} / I_{\mathrm{G}}\right){ }^{22}$ The $R$ values of the CNFM-0 and CNFM-20 are 1.51 and 1.04, respectively. The low $R$ value of the CNFM-20 confirms that addition of PSF improves the degree of carbonization in CNFM-20. The improved degree of carbonization and the interconnected nanofibrous morphology (Fig. 1b') results in high conductivity of CNFM-20, as shown in Table 1 . Fig. S4 $\dagger$ shows the XPS characterization of the surface chemical compositions of the CNFMs. The surfaces of both CNFM-0 and CNFM-20 contain three elements, C, N, and O. The PSF/PAN based CNFM-20 has relatively high $\mathrm{C}$ contents compared to the PAN based CNFM-0. The percentage of each element is shown in the inset of Fig. S4. $\uparrow$ CNFM-20 has the higher carbon percentage $(94.7 \%)$ than CNFM-0 (91.5\%).

The thermal behaviors of the CNFMs are investigated by TGA and DSC analysis. Fig. 2d shows the TGA curves of PSF, the PAN nanofibrous mat, and the PSF/PAN nanofibrous mat. The pristine PSF is stable with no weight loss below about $500{ }^{\circ} \mathrm{C}$. It degrades quickly when the temperature exceeds $500{ }^{\circ} \mathrm{C}$ and loses about $60 \%$ of the weight from 500 to $600{ }^{\circ} \mathrm{C}$; above $600{ }^{\circ} \mathrm{C}$, the remaining PSF only loses small percentage of its weight, leaving about $32 \%$ of carbon residue. The result suggests that PSF decomposes completely between 500 to $600{ }^{\circ} \mathrm{C}$ and it is carbonized at relatively low temperature. The PAN nanofibrous mat starts to lose weight significantly above the pre-oxidation temperature of $280{ }^{\circ} \mathrm{C}$; the weight loss slows down above $\sim 500{ }^{\circ} \mathrm{C}$ but continues during the carbonization process. The weight loss of the PSF/PAN nanofibrous mat is less than that of the PAN nanofibrous mat below $\sim 500{ }^{\circ} \mathrm{C}$; above $500{ }^{\circ} \mathrm{C}$, because of the quick decomposition of PSF, the PSF/PAN nanofibrous mat loses weight quickly. Eventually, the PSF/PAN nanofibrous mat has a slightly lower carbon yield in comparison with the PAN mat. Fig. S5 $\uparrow$ shows the result of DSC analysis of the pre-oxidation and 
Table 1 Surface areas, pore parameters, conductivity, and capacitance of CNFM-0 and CNFM-20

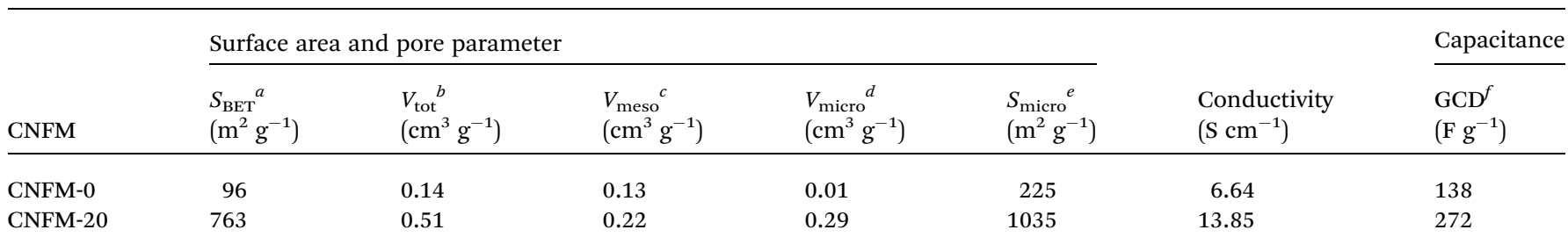

${ }^{a} S_{\mathrm{BET}}$ : specific surface area by BET. ${ }^{b} V_{\text {tot }}$ : total pore volume, measured at $P / P_{0}=0.995 .{ }^{c} V_{\text {meso }}$ : mesopore volume by BJH. ${ }^{d} V_{\text {micro }}$ : micropore volume by Langmuir. ${ }^{e} S_{\text {micro }}$ specific surface area by Langmuir. ${ }^{f}$ Measured by galvanostatic charge/discharge (GCD) at $1.0 \mathrm{~A} \mathrm{~g}^{-1}$.

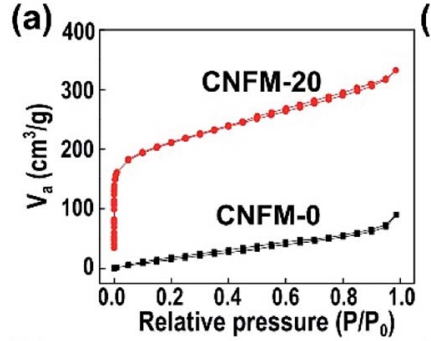

(c)
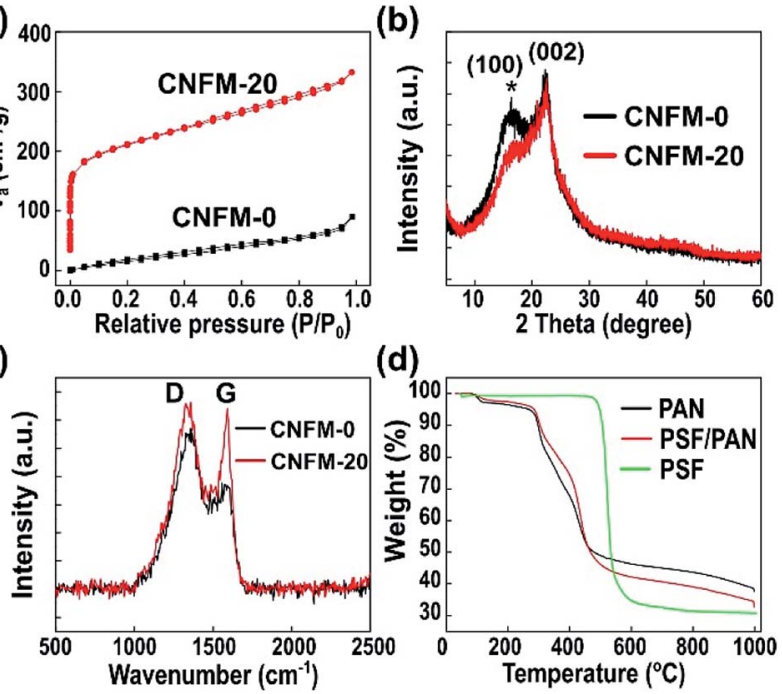

(d)

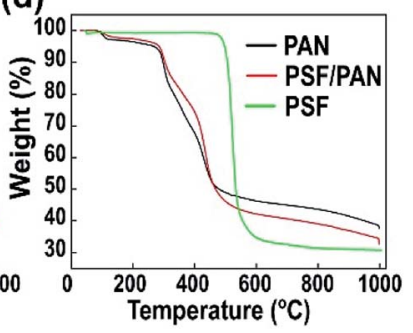

Fig. 2 (a) $\mathrm{N}_{2}$ adsorption/desorption isotherms of CNFM-O and CNFM20. (b) XRD patterns of CNFM-O and CNFM-20. The graphitic crystallite plane (002) and the PAN crystallite plane (100) are labelled. (c) Raman spectra of CNFM-O and CNFM-20. (d) TGA curves of PAN nanofibers, PSF/PAN nanofibers, and PSF.

carbonization process for preparation of both CNFM-0 and CNFM-20. The DSC curve of PAN nanofibrous mat has a pronounced exothermic peak at about $285{ }^{\circ} \mathrm{C}$ (due to preoxidation) and two broad exothermic peaks during carbonization. ${ }^{23}$ The DSC curve of the PSF polymer shows a glass transition temperature of $\sim 200{ }^{\circ} \mathrm{C}$ and a more pronounced endothermic transition at $\sim 500{ }^{\circ} \mathrm{C}$, in consistence with decomposition of PSF observed in TGA measurement (Fig. 2d). It is worth noting that PSF typically has a melt processing temperature of $\sim 350{ }^{\circ} \mathrm{C} .{ }^{24}$ Therefore, PSF may be in a low-viscosity melting state in the preoxidized CNFM-20 before decomposition at above $500{ }^{\circ} \mathrm{C}$. As a result, the melted PSF polymer facilitates the formation of fibrous network of CNFM-20.

The interconnected fibrous network (Fig. 1 $\mathrm{b}^{\prime}$ ) and the high content of graphitic carbon (as suggested by Fig. $2 \mathrm{~b}$ and c) of CNFM-20 lead to high conductivity of the material. As shown in Table 1, the conductivity of PAN-derived CNFM-0 is $6.64 \mathrm{~S} \mathrm{~cm}^{-1}$; and the conductivity of PSF/PAN-derived CNFM-20 is $13.86 \mathrm{~S} \mathrm{~cm}^{-1}$, about twice that of CNFM-0.

Based on the XRD, Raman, XPS, and thermal analysis results, the high surface areas, hierarchical porous structures, good electric conductivity of CNFM-20 can be explained by the chemical and structural changes during pre-oxidation and carbonization process, as shown in Fig. 3. For CNFM-0 derived from the PAN nanofibers, it is well known that the stable cyclic ladder molecule structure and the six membered ring graphite structure are obtained during pre-oxidation and carbonization steps, respectively. ${ }^{25}$ As shown in Fig. 3a, no interconnection at the fiber junction points is formed, presumably because the stable cyclic ladder molecule structure during the pre-oxidation prevents melting/softening of PAN nanofibers to form connected junctions. In addition, the CNFM prepared from pure PAN nanofibers typically have smooth surface, resulting in small number of mesopores and micropores and low specific surface area. The formation of CNFM-20 from the PSF/PAN nanofibers is schematically shown in Fig. $3 \mathrm{~b}$. During the preoxidation process, similar formation of stable cyclic ladder molecule structure of PAN is expected with no chemical change of PSF because of the thermal stability of PSF at $280^{\circ} \mathrm{C}$. The PSF component in PSF/PAN becomes soft above the glass transition temperature $\left(\sim 200{ }^{\circ} \mathrm{C}\right)$ and is further softened/melt at higher temperature before decomposition. After decomposition, the PSF molecular chains continue being carbonized with increase

(a)

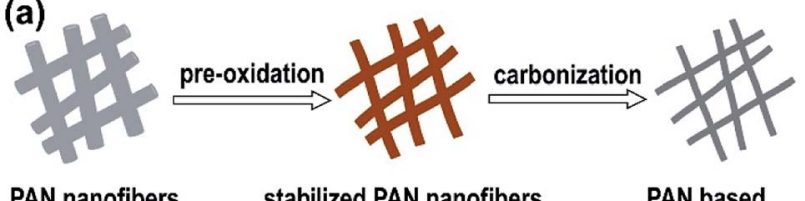

PAN nanofibers

stabilized PAN nanofibers

PAN based (b)
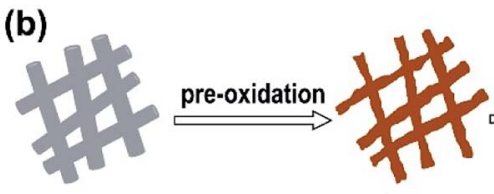

PSFIPAN nanofibers stabilized PSF/PAN nanofibers carbon nanofibers

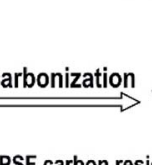

PSF carbon residu

PSFIPAN based carbon nanofibers

(c)

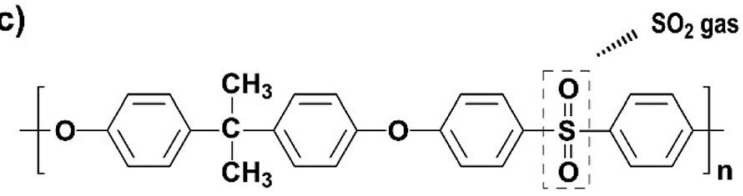

Fig. 3 (a) Schematic illustration of formation of the PAN based CNFM0 . (b) Schematic illustration of formation of the PSF/PAN based CNFM20. (c) The chemical structure of the repeating unit of PSF and the chemical change during decomposition. 
of the temperature. Since the PSF polymer chains are broken into short chains, these shortened chains would further act as a glue at fiber junction points to fuse the carbonized PSF/PAN nanofibers together during decomposition and carbonization. Moreover, the decomposition of PSF in the carbon nanofibers leads to formation of mesopores, which is an important factor for increase of the specific surface area in CNFM-20. As shown in Fig. 3c, the repeating unit of PSF contains four benzene rings and a sulfonate group, thus PSF has a high thermal degradation temperature of approximately $550{ }^{\circ} \mathrm{C}$; furthermore, the sulfone group of the PSF molecule is released as the $\mathrm{SO}_{2}$ gas from the nanofibers. ${ }^{26-28}$ The release of $\mathrm{SO}_{2}$ contributes to the formation of micropores, increasing the Langmuir surface area in the PSF/ PAN derived CNFM-20 (as shown in Table 1).

The electrochemical performance of the CNFMs is investigated in a three-electrode configuration. The inset of Fig. 4a shows the images of CNFM-20 pressed directly on the nickel foam electrode. Fig. 4a shows the CV curves of both CNFM0 and CNFM-20 at a scan rate of $10 \mathrm{mV} \mathrm{s}{ }^{-1}$. Both CNFMbased electrodes have a near-rectangular shape, indicating excellent EDLC behavior and low internal resistance. The slight increase of current below $-0.8 \mathrm{~V}$ may be due to the pseudocapacitive contribution from the $\mathrm{N}$ and/or $\mathrm{O}$ groups in CNFMs (Fig. S4 $\dagger$ ), which is common in the porous carbon electrode materials. ${ }^{29}$ The CV curve of CNFM-20 has larger rectangular area compared to that of CNFM-0, indicating higher capacitance of CNFM-20.

Fig. $4 \mathrm{~b}$ shows the GCD behavior of the CNFMs at a current density of $1.0 \mathrm{~A} \mathrm{~g}^{-1}$ in a three-electrode configuration and $2.0 \mathrm{M}$ $\mathrm{KOH}$ electrolyte. The GCD curves of the CNFMs at different current densities are shown in Fig. S6. $\uparrow$ The triangular shape of the GCD curves at various current densities indicates efficient
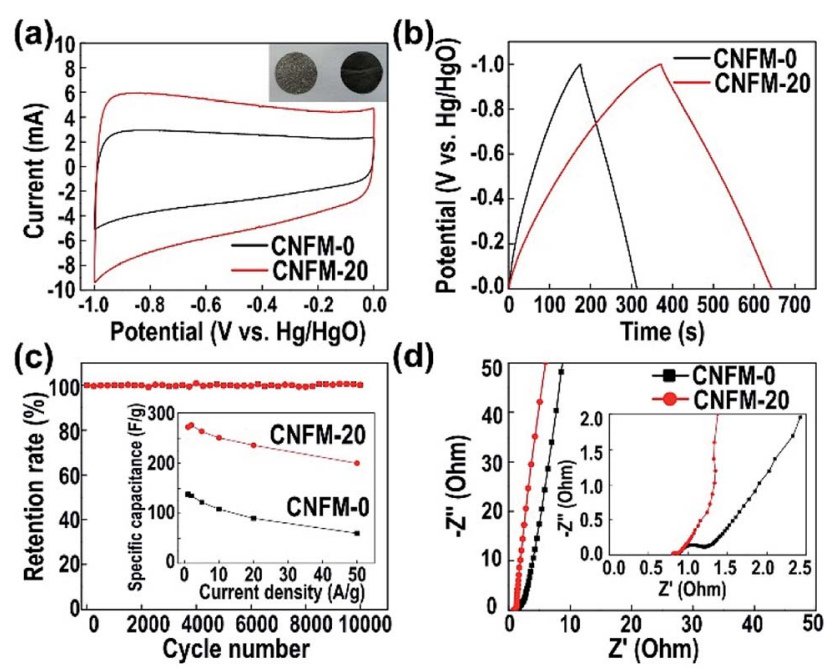

Fig. 4 (a) CV curves of CNFM-O and CNFM-20. Inset: the images of the nickel foam (left) and the CNFM-20 nanofibrous mat pressed on the nickel foam (right). (b) GCD curves of CNFM-0 and CNFM-20 at current density of $1.0 \mathrm{~A} \mathrm{~g}^{-1}$. (c) Cyclic performance of CNFM-O and CNFM-20 measured at current density of $1.0 \mathrm{~A} \mathrm{~g}^{-1}$. Inset: specific capacitances of CNFM-O and CNFM-20 at current densities from $1.0 \mathrm{~A} \mathrm{~g}^{-1}$ to $50 \mathrm{~A} \mathrm{~g}^{-1}$. (d) Nyquist plots of the impedance of CNFM0 and CNFM-20. ionic and electronic transport and good EDLC formation in both CNFM electrodes. CNFM-20 prepared from the PSF/PAN nanofibers have longer discharge time than the PAN based CNFM-0; the result is consistent with the higher capacitance in CNFM-20 observed in the CV measurement. The specific capacitance of the electrode (listed in Table 1) is calculated using the following eqn (1) from the GCD curve. ${ }^{30}$

$$
C_{\mathrm{s}}=\frac{I \times \Delta t}{m \times \Delta V}
$$

where $C_{\mathrm{s}}\left(\mathrm{F} \mathrm{g}^{-1}\right)$ is the specific capacitance, $\Delta V(\mathrm{~V})$ is the potential change within the discharge time $\Delta t(\mathrm{~s}), m(\mathrm{~g})$ is the mass of the active materials, and $I$ (A) is the set current of the GCD measurement. The CNFM-20 electrode has a specific capacitance of $272 \mathrm{~F} \mathrm{~g}^{-1}$, while the CNFM-0 electrode shows a specific capacitance of $138 \mathrm{~F} \mathrm{~g}^{-1}$. The GCD specific capacitances of both CNFMs at charging rate from $1 \mathrm{~A} \mathrm{~g}^{-1}$ to $50 \mathrm{~A} \mathrm{~g}^{-1}$ are shown in the inset of Fig. 4c. At a high current density of $50 \mathrm{Ag}^{-1}$, the CNFM-20 and CNFM-0 electrodes retain $74 \%$ and $44 \%$ of the specific capacitances at the current density of $1.0 \mathrm{~A} \mathrm{~g}^{-1}$, respectively. The coulombic efficiency, defined as the ratio of discharge time and charge time at the same current density, for the electrode of CNFM-20 is $80 \%$, whereas the coulombic efficiency for the electrode of CNFM-0 is $73 \%$. The high retention rate and coulombic efficiency of the CNFM-20 electrode indicates the excellent supercapacitor performance of the materials, which originates from the high surface areas, hierarchical porous structure and interconnected carbon nanofiber network of CNFM-20. To further evaluate the cycle performance, the CNFM electrodes in three-electrode configuration are charged and discharged repeatedly for 10000 cycles at a current density of $1.0 \mathrm{~A} \mathrm{~g}^{-1}$ in 2.0 M KOH electrolyte. As shown in Fig. 4c, after 10000 cycles of charge and discharge at $1.0 \mathrm{~A} \mathrm{~g}^{-1}$, the specific capacitance retention rates for both CNFM electrodes are nearly $100 \%$, indicating excellent cycling stability of the CNFMs. The GCD curves of the CNFM-20 electrode at the $1^{\text {st }}$ and the $10000^{\text {th }}$ cycle are shown in Fig. S7. $\dagger$ Overall, the electrochemical characterization results show that CNFM-20 is an excellent electrode material for supercapacitor. The hierarchical pore structures provide a larger specific surface area for CNFM-20, which leads to the adsorption of more ions in the electrode; the interconnected fibrous structure and excellent conductivity also accelerate the transport of electrons. As a result, the synergistic effect of larger specific surface area, interconnected fibrous structure and excellent conductivity leads to high specific capacitance during charge and discharge process for the CNFM-20 electrode. Table S1 $\uparrow$ summarizes the surface properties and the supercapacitor performance of the electrospun CNFMs reported in recent years. In comparison with CNFMs prepared by other methods, the CNFMs prepared by carbonization of electrospun PSF/PAN nanofibrous mat in our work show superior surface properties and EDLC performance. Additionally, the PSF/PAN-derived CNFM-20 does not require post-preparation activation, while most of the methods in the literature require activation to form large surface areas and pores.

To further understand the electronic and ion transport in CNFMs, EIS measurements are carried out. The Nyquist plots of 
CNFM-20 and CNFM-0 are shown in Fig. 4d. The result shows a typical behavior of EDLCs based on porous carbon electrode. As shown in the inset of Fig. 4d, the semicircle at high frequency represents the accessibility of the pores (micropores/ mesopores) to the ions in the electrolyte (referred to as the charge transfer resistance, $\left.R_{\mathrm{ct}}\right),{ }^{31,32}$ and the intercept at the real axis represents the equivalent series resistance $\left(R_{\mathrm{s}}\right)$, whereas the linear behavior at the low frequency reflects the ion diffusion and capacitive response (called Warburg resistance). The large slope of the linear region of CNFM-20 indicates lower ion diffusion resistance and better capacitive behavior in comparison with CNFM-0. The smaller semicircle in the Nyquist plot of CNFM-20 in comparison to that of CNFM-0 indicates smaller $R_{\mathrm{ct}}$ in the CNFM-20 electrode, which can be attributed to the fact that the hierarchical pore structures of CNFM-20 result in better ion accessibility. Additionally, $R_{\mathrm{s}}$ of the CNFM-20 electrode is $0.8 \Omega$, which is smaller than $R_{\mathrm{s}}$ of the CNFM-0 electrode $(2.2 \Omega)$, in consistent with the better conductivity of CNFM-20. Furthermore, the frequency response of the CNFMs is explored from the Bode plots (as shown in Fig. S8†), and the time constant $t_{0}$, which is defined as $1 / f_{0}$ and reflects how fast the electrode can be reversibly charged and discharged under the characteristic frequency $f_{0}$ at $-45^{\circ}$ phase, can be calculated. The CNFM-20 electrode has a shorter time constant $\left(t_{0}=1.0 \mathrm{~s}\right)$ than the CNFM-0 electrode $\left(t_{0}=1.43 \mathrm{~s}\right)$, in consistent with a better rate handling ability of CNFM-20.

The excellent electrochemical performance of the CNFMs prepared by electrospinning and carbonization of PAN with polysulfone as a sacrificial polymer enables us to make twoelectrode supercapacitor without carbon activation and electrode additives (e.g. PVDF and active carbon). The CV curves of the device (Fig. 5a) at a scan rate ranging from $10 \mathrm{mV} \mathrm{s}^{-1}$ to $500 \mathrm{mV} \mathrm{s}^{-1}$ have symmetric, rectangular shape; at a scan rate as high as $500 \mathrm{mV} \mathrm{s}^{-1}$, the $\mathrm{CV}$ curve remains its rectangular shape,

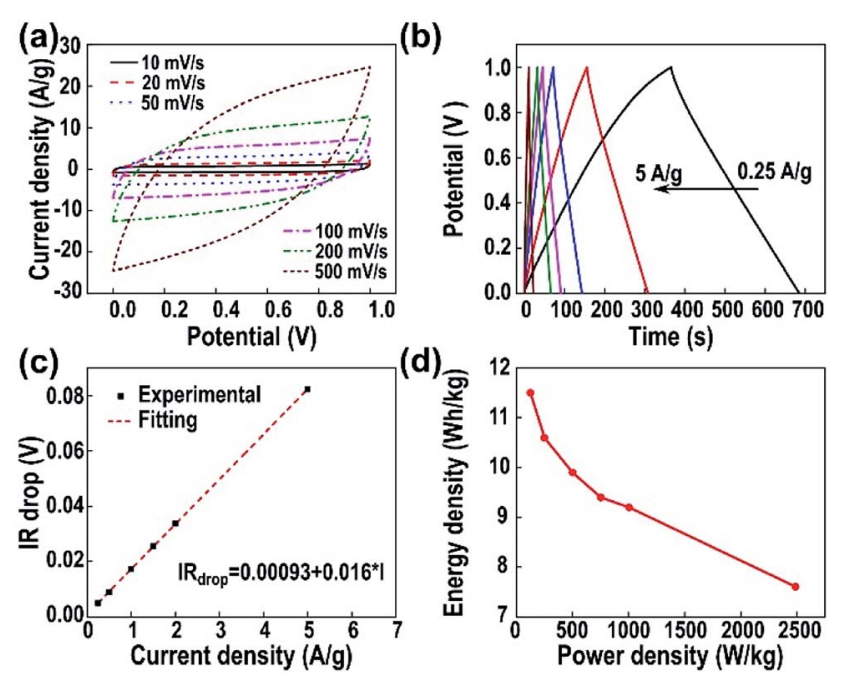

Fig. 5 (a) CV curves of the two-electrode supercapacitor based on the freestanding CNFM-20 nanofibrous mat. (b) GCD curves of the CNFM20 based device at current densities ranging from $0.25 \mathrm{~A} \mathrm{~g}^{-1}$ to $5 \mathrm{~A} \mathrm{~g}^{-1}$ (c) IR drop of the device vs. the current density. (d) The Ragone plot of energy density and power density of the CNFM-20 based supercapacitor. indicating excellent capacitive performance. Fig. 5b shows the GCD curves of the CNFM-20 based supercapacitor charged/ discharged with different current density. The linear and symmetric GCD curves show that the CNFM-20 based device has excellent reversible behavior and ideal electric double-layer capacitive characteristics under fast charge/discharge. Additionally, the GCD curves with various charge/discharge rates have very small IR drop. Plot of the IR drop vs. current density in Fig. $5 c$ shows a linear relationship, which can be fit based on the following eqn (2). ${ }^{33}$

$$
\mathrm{IR}_{\mathrm{drop}}=a+b I
$$

here, $a$ represents the difference between the applied potential of $1.0 \mathrm{~V}$ and the charged potential of the capacitor, $b$ represents twice the value of the internal resistance, and $I$ (A) is the discharge current. The small values of $a$ and $b$ for the linear fitting in Fig. $5 \mathrm{c}$ is consistent with the good performance of the device, which may be attributed to the hierarchically porous and interconnected structure of CNFM-20. The maximum power density $\left(P_{\max }\right)$ of the device is calculated to be $30.7 \mathrm{~kW} \mathrm{~kg}^{-1}$ by the following eqn (3). ${ }^{33}$

$$
P_{\max }=\frac{V_{0}^{2}}{4 \times M \times R_{\mathrm{s}}}=\frac{(1-a)^{2}}{2 \times M \times b}
$$

where $V_{0}(\mathrm{~V})$ is the practical working potential of the supercapacitor device.

Fig. 5d shows the Ragone plot of energy density and power density of the CNFM-20 based supercapacitor. The power density $(P)\left(\mathrm{W} \mathrm{kg}^{-1}\right)$ and the energy density $(E)\left(\mathrm{W} \mathrm{h} \mathrm{kg}^{-1}\right)$ of a supercapacitor are calculated using following eqn (4)-(6): ${ }^{30}$

$$
\begin{gathered}
C_{\mathrm{m}}=\frac{I \times \Delta t}{M \times \Delta V} \\
E=\frac{1}{2} \times C_{\mathrm{m}} \times(\Delta V)^{2} \\
P=\frac{E}{\Delta t}
\end{gathered}
$$

where $C_{\mathrm{m}}\left(\mathrm{F} \mathrm{g}^{-1}\right)$ is the specific capacitance of device, $\Delta V(\mathrm{~V})$ is the potential change within the discharge time $\Delta t(\mathrm{~s}), M(\mathrm{~g})$ is the mass of the active materials in the two electrodes, and $I$ (A) is the set current of the GCD measurement. The energy density decreases with increase of power density. The supercapacitor device based on the CNFM-20 electrodes has the maximum energy density of $11.5 \mathrm{~W} \mathrm{~h} \mathrm{~kg}^{-1}$ and maximum power density of $2.49 \mathrm{~kW} \mathrm{~kg}^{-1}$. These values are comparable with those of supercapacitors based on carbon nanomaterials. ${ }^{34}$

\section{Conclusion}

In conclusion, carbon nanofibrous mats with high surface areas, hierarchical porous structures, and interconnected fibrous network are prepared by carbonization of electrospun nanofibers of PAN and PSF blend. The rapid degradation of PSF between 500 to $600{ }^{\circ} \mathrm{C}$ accompanied by release of $\mathrm{SO}_{2}$ gas results in formation of micropores and mesopores. Softened PSF at 
temperatures above the pre-oxidation $\left(280{ }^{\circ} \mathrm{C}\right)$ and the high carbon residue after PSF degradation facilitate formation of interconnected junctions in the nanofibrous mat during carbonization of PSF/PAN. Additionally, the amount of graphitic carbon in the PSF/PAN based carbon nanofibrous mat increases in comparison to that in the PAN based carbon nanofibrous mat. These desired structural properties lead to high conductivity and excellent electrochemical performance of the PSF/PAN based carbon nanofibrous mat for supercapacitor electrode. The electrode from the PSF/PAN based carbon nanofibrous mat shows a specific capacitance of $272 \mathrm{~F} \mathrm{~g}^{-1}$ at current density of $1 \mathrm{~A} \mathrm{~g}^{-1}$, excellent cycling durability $(100 \%$ capacitance retention after 10000 cycles), and good rate retention at current density of $50 \mathrm{~A} \mathrm{~g}^{-1}$.

\section{Conflicts of interest}

There are no conflicts to declare.

\section{Acknowledgements}

This work is supported by the National Natural Science Foundation of China (Grant Number 51573136), the Natural Science Foundation of Tianjin (Grant Number 15JCYBJC17800), and the Science and Technology Plans of Tianjin (Grant Number 16PTSYJC00110).

\section{Notes and references}

1 M. Winter and R. J. Brodd, Chem. Rev., 2004, 104, 4245-4270.

2 P. Simon and Y. Gogotsi, Nat. Mater., 2008, 7, 845-854.

3 G. Wang, L. Zhang and J. Zhang, Chem. Soc. Rev., 2012, 41, 797-828.

4 A. Borenstein, O. Hanna, R. Attias, S. Luski, T. Brousse and D. Aurbach, J. Mater. Chem. A, 2017, 5, 12653-12672.

5 F. Wang, X. Wu, X. Yuan, Z. Liu, Y. Zhang, L. Fu, Y. Zhu, Q. Zhou, Y. Wu and W. Huang, Chem. Soc. Rev., 2017, 46, 6816-6854.

6 C. Lai, Z. Zhou, L. Zhang, X. Wang, Q. Zhou, Y. Zhao, Y. Wang, X.-F. Wu, Z. Zhu and H. Fong, J. Power Sources, 2014, 247, 134-141.

7 X. Lu, C. Wang, F. Favier and N. Pinna, Adv. Energy Mater., 2017, 7, 1601301.

8 B.-H. Kim, K. S. Yang and J. P. Ferraris, Electrochim. Acta, 2012, 75, 325-331.

9 L. Zhang, A. Aboagye, A. Kelkar, C. Lai and H. Fong, J. Mater. Sci., 2014, 49, 463-480.

10 C. Kim and K. S. Yang, Appl. Phys. Lett., 2003, 83, 1216-1218.

11 Y.-W. Ju, S.-H. Park, H.-R. Jung and W.-J. Lee, J. Electrochem. Soc., 2009, 156, A489-A494.
12 T. Le, Y. Yang, Z. Huang and F. Kang, J. Power Sources, 2015, 278, 683-692.

13 K. Huang, M. Li, Z. Chen, Y. Yao and X. Yang, Electrochim. Acta, 2015, 158, 306-313.

14 N. C. Abeykoon, J. S. Bonso and J. P. Ferraris, RSC Adv., 2015, 5, 19865-19873.

15 Y.-H. Hsu, C.-C. Lai, C.-L. Ho and C.-T. Lo, Electrochim. Acta, 2014, 127, 369-376.

16 Y. Yao, K. K. Fu, S. Zhu, J. Dai, Y. Wang, G. Pastel, Y. Chen, T. Li, C. Wang, T. Li and L. Hu, Nano Lett., 2016, 16, 72827289.

17 J. Ding, H. ping Zhang, X. Li, Y. Tang and G. Yang, Mater. Des., 2018, 141, 17-25.

18 H. Wang, W. Wang, H. Wang, X. Jin, H. Niu, H. Wang, H. Zhou and T. Lin, ACS Appl. Energy Mater., 2018, 1, 431439.

19 Z. Zhou, C. Lai, L. Zhang, Y. Qian, H. Hou, D. H. Reneker and H. Fong, Polymer, 2009, 50, 2999-3006.

20 M. Wu, Q. Wang, K. Li, Y. Wu and H. Liu, Polym. Degrad. Stab., 2012, 97, 1511-1519.

21 A. M. Al-Enizi, A. A. Elzatahry, A. M. Abdullah, M. A. AlMaadeed, J. Wang, D. Zhao and S. Al-Deyab, Carbon, 2014, 71, 276-283.

22 D. S. Knight and W. B. White, J. Mater. Res., 1989, 4, 385-393. 23 Y.-X. Wang, C.-G. Wang, J.-W. Wu and M. Jing, J. Appl. Polym. Sci., 2007, 106, 1787-1792.

24 https:/www.solvay.com/en/markets-and-products/featuredproducts/udel.html, (last accessed: February 4, 2018).

25 M. Rahaman, A. Ismail and A. Mustafa, Polym. Degrad. Stab., 2007, 92, 1421-1432.

26 P. Almén and I. Ericsson, Polym. Degrad. Stab., 1995, 50, 223228.

27 L. Perng, J. Polym. Sci., Part A: Polym. Chem., 2000, 38, 583593.

28 S. T. Ellison, A. P. Gies, D. M. Hercules and S. L. Morgan, Macromolecules, 2009, 42, 3005-3013.

29 L. Qie, W. Chen, H. Xu, X. Xiong, Y. Jiang, F. Zou, X. Hu, Y. Xin, Z. Zhang and Y. Huang, Energy Environ. Sci., 2013, 6, 2497.

30 S. Zhang and N. Pan, Adv. Energy Mater., 2014, 5, 1401401. 31 J. Kang, J. Wen, S. H. Jayaram, X. Wang and S.-K. Chen, J. Power Sources, 2013, 234, 208-216.

32 A. Allagui, T. J. Freeborn, A. S. Elwakil and B. J. Maundy, Sci. Rep., 2016, 6, 38568.

33 A. Izadi-Najafabadi, S. Yasuda, K. Kobashi, T. Yamada, D. N. Futaba, H. Hatori, M. Yumura, S. Iijima and K. Hata, Adv. Mater., 2010, 22, E235-E241.

34 T. Liu, F. Zhang, Y. Song and Y. Li, J. Mater. Chem. A, 2017, 5, 17705-17733. 